\title{
Vers de nouvelles études en thermohydraulique diphasique pour les applications industrielles
}

\author{
Towards new studies in two-phase thermal-hydraulics \\ for industrial applications
}

par O. Lebaigue et E. Hervieu

Commissariat à l'Energie Atomique, Grenoble

This paper gives a short presentation of the FASTNET team preliminary conclusions. This team tried to define a scientific strategy aimed at developing thermal-hydraulics in the nuclear and non-nuclear industry fields. The reflections were mainly conducted as a three stage process :

- the identification of industrial problems, whose better resolution rely on a knowledge improvement in thermalhydraulics,

- a state-of-the-art analysis, which led to identify the actual limitations (and their origin) for the different components of the thermal-hydraulics expertise (physical modeling, numerical simulation, instrumentation, analytical and validation experiments),

- the proposition of a coherent strategy to develop expertise in all these fields of thermal-hydraulics.

This paper is a summarized version of a more detailed progress report. The work is still in progress...

\section{INTRODUCTION}

Le groupe de travail FASTNET (Force d'Analyse Scientifique et Technique pour de Nouvelles Etudes en Thermohydraulique) a tenté de définir une stratégie scientifique à dix ans pour le développement de la thermohydraulique appliquée aux domaines nucléaire et non nucléaire de l'industrie en relation avec les activités du CEA. Les réflexions de ce groupe l'ont successivement amené à identifier les problèmes industriels pour lesquels le potentiel de progrès est industriellement motivant et repose en particulier sur un approfondissement des connaissances en thermohydraulique.

L'étape suivante de l'analyse a consisté à dresser un état de l'art des recherches de base entreprises en France et à l'étranger, en insistant particulièrement sur l'identification des limitations actuelles concernant :

- la modélisation des phénomènes physiques,

- les techniques numériques associées à la simulation,

- les techniques de mesure,

- les techniques expérimentales.

Enfin le groupe a commencé à construire une stratégie de recherche visant à lever les principaux obstacles identifiés. Il s'agit là d'un programme innovant et ambitieux, dont les enjeux dépassent le champ d'action habituel du CEA mais aussi ses moyens propres. Pour mener à bien une bonne part des recherches recommandées, il serait préférable d'associer aux différents départements du CEA concernés, outre ses partenaires industriels habituels, d'autres intervenants concernés par les progrès potentiels à réaliser dans le domaine de la thermohydraulique.

La présente évocation, réduite à quelques pages, va tenter de reprendre l'essentiel de l'articulation de la réflexion concernant la thermohydraulique diphasique et polyphasique. Cependant, il ne s'agit que d'un extrait de documents plus volumineux, rendant compte d'une réflexion en cours $[1,2]$. En outre, cette réflexion n'est pas l'apanage du groupe FASTNET, et s'élargit au niveau du CEA notamment par des contacts formels (e.g. contacts avec l'Advanced Thermal Hydraulic Research Institute, financé par la NRC américaine) ou informels, tels ceux réalisés au travers de structures ou réseaux comme le GREDIC (GDR 1205 du CNRS).

\section{II — PROBLÈMES INDUSTRIELS NON RÉSOLUS}

\subsection{Les approches actuelles des problèmes industriels}

Parmi tous les problèmes industriels de thermohydraulique monophasique ou polyphasique, il est difficile d'en trouver pour lesquels aucune réponse ne peut être donnée. Généralement, pour analyser le problème, une ou plusieurs expériences sont nécessaires. Très souvent, le recours à des codes de thermohydraulique est indispensable. Des itérations entre codes et expériences sont souvent utiles. Les expériences 
servent à analyser le problème, à développer des modèles pour les codes, puis à qualifier ces codes.

Les codes peuvent servir à des études préliminaires pour concevoir les expériences, peuvent aider à interpréter les expériences, et sont souvent nécessaires comme outils d'interpolation ou d'extrapolation pour passer des résultats de l'expérience au problème industriel.

Ces méthodes d'approche des problèmes industriels sont souvent lourdes et difficiles à mettre en œuvre. Les principales difficultés sont les suivantes :

- la réponse au problème peut être insuffisamment fiable et l'incertitude sur la réponse est difficile à estimer,

- les délais d'obtention d'une réponse peuvent être très longs,

- le coût total des études expérimentales et numériques peut être très élevé.

\section{- 2.2 Les spécificités de la thermohydraulique diphasique}

La thermohydraulique diphasique possède les caractéristiques suivantes :

- grande variété et complexité des mécanismes physiques et des configurations d'écoulement,

- nombreux paramètres physiques et nombres sans dimension,

- insuffisance des techniques de mesure pour appréhender l'ensemble des processus,

- compréhension insuffisante de nombreux phénomènes physiques de base.

Dans ce contexte, très peu de problèmes industriels peuvent être résolus sans recours à l'expérience, laquelle est souvent nécessaire à l'échelle 1. Bien que, comme en écoulements monophasiques, les relations de bilans dans chaque phase (Navier-Stokes) et aux interfaces soient connues, les codes existants sont très loin de la simulation directe, sauf dans des cas où la géométrie est très simple. Ces codes ont recours à des approches moyennées nécessitant de nombreuses relations de fermeture qui ont un caractère plus ou moins empirique. Par conséquent, ces codes requièrent un important programme de validation dans tout le domaine utile. Ils fonctionnent aujourd'hui assez bien en interpolation, mais sont insuffisamment fiables en extrapolation. De plus, les situations fortement tridimensionnelles souffrent d'un manque d'outils validés à la fois sur le plan de la modélisation et sur le plan numérique, en dehors de quelques configurations d'écoulement (écoulements dispersés dilués et à fines inclusions). Enfin, les méthodes numériques utilisées en diphasique sont robustes mais souvent peu précises et diffusives. Par conséquent, de nombreux problèmes peuvent être résolus par une combinaison code-expérience de qualification mais avec des coûts et délais relativement importants et le travail est à recommencer dès que l'on change de fluide, de géométrie, de gamme de paramètres physiques,... Dans le cas de certains régimes d'écoulement, des problèmes typiquement multidimensionnels n'ont pas d'outil numérique adapté.

\subsection{Exemples de problèmes industriels non ou mal résolus}

Nous avons listé ici des familles de problèmes pour lesquels l'amélioration des connaissances en thermohydraulique est une source potentielle de progrès, car, bien que l'ingénieur ait généralement déjà trouvé une solution d'urgence au cas par cas, ces problèmes requièrent encore des solutions générales, fiables et validées. Cette liste concerne le domaine nucléaire, mais également le génie pétrolier, le génie chimique,... :

- définition et caractérisation des régimes d'écoulement, prédiction de leur transition,

- caractéristiques hydrauliques d'un circuit en diphasique : pertes de pression, détermination de taux de présence,

- détermination du flux thermique critique,

- ébullition en film inversé (e.g. lors du renoyage de crayons de combustible nucléaire), température minimum du film stable,

— sollicitations vibratoires des cintres de générateurs de vapeur,

- trempe de matériaux,

- écoulements diphasiques avec blocage sonique,

- limites d'engorgement dans des géométries complexes,

- problèmes de condensation par contact direct (sprays, injecteurs,...),

- dimensionnement et performances d'échangeurs en régime diphasique,

- entraînement de gaz dans un écoulement à surface libre, - performances de composants complexes : séparateurs, sécheurs, pompes diphasiques,...

- mélange de fluides non miscibles,

- désorption nucléée en paroi de gaz dissous,

- explosions de vapeur.

\section{III — LIMITATIONS ACTUELLES IDENTIFIÉES}

\subsection{Limitations concernant la modélisation physique}

Dans l'état de l'art actuel, la modélisation physique des écoulements diphasiques est le plus souvent représentée par des approches moyennées mono- ou multidimensionnelles, mais on commence à voir se développer des approches plus fines, qui sont l'analogue pour le diphasique des approches monophasiques de type LES. Il existe également des tentatives de simulation directe avec suivi de chaque interface.

Les approches monodimensionnelles moyennées sont de loin les plus développées et sont aujourd'hui couramment utilisées dans des codes industriels. Les équations sont moyennées sur la section de la conduite et dans le temps, ce qui permet d'oublier la structure fine des écoulements et tous les phénomènes liés à l'intermittence diphasique et aux fluctuations turbulentes. Seuls les aspects macroscopiques sont visés. Leur performance repose sur le choix du nombre de paramètres descriptifs de l'écoulement (modèles homogènes, modèles à 4 ou 5 équations, modèles à deux fluides et 6 équations, modèles multi-champs), et sur la validité des relations de fermeture (qui dépasse très rarement le domaine de construction). Les modèles à deux fluides peuvent rendre compte de tous les déséquilibres thermique et mécanique entre phases (glissement entre phases, liquide sous-saturé, vapeur surchauffée, états métastables,...) pour toutes les configurations d'écoulement. Les modèles multi-champs deviennent nécessaires lorsqu'une phase est présente dans deux ou plusieurs champs faiblement couplés (film liquide et champ de gouttes). Les limitations communes à tous ces modèles monodimensionnels apparaissent lorsque les processus physiques à petite échelle (fractionnement, coalescence, stratification, ...) qui sont pris en compte dans les relations de fermeture ont des temps de relaxation significatifs. Leur prise en compte nécessiterait de rajouter des équations de 
transport de grandeurs caractéristiques de la structure des interfaces telles que l'aire interfaciale volumique. Une limitation plus générale réside dans le manque d'un accès commun et didactique aux différents modèles.

Le besoin de décrire des géométries particulières compliquées impose l'approche multidimensionnelle qui, seule, permet de progresser dans la description fine des écoulements diphasiques. Cette approche est assez avancée aujourd'hui pour les écoulements dispersés dilués. Les efforts ont porté sur la modélisation des effets de la turbulence de la phase continue sur la dispersion des inclusions. Du fait de la petite taille des inclusions, les perturbations du champ turbulent sont limitées aux grands nombres d'onde et les grosses structures turbulentes responsables de la diffusion sont peu affectées. En revanche, cela n'est pas le cas pour les écoulements diphasiques très déstructurés à grosses poches distordues (churn flow) : les modèles moyennés actuels ne traitent que les cas où la turbulence gouverne le diphasique mais ils ignorent encore les cas où le diphasique gouverne la turbulence. Dans le cas de l'approche multidimensionnelle également, l'ajout d'une équation de transport de l'aire interfaciale volumique permettrait de tenir compte de la configuration locale de l'écoulement [3].

Il existe cependant des approches plus fines pour les écoulements dispersés dilués et à fines inclusions où le filtrage des fluctuations turbulentes ne concerne que les plus petites échelles. Ces approches sont analogues aux modèles monophasiques de type LES. Elles ne sont valables que lorsque le temps d'interaction entre chaque particule et le fluide porteur est inférieur à l'échelle de temps du filtrage de la turbulence de la phase continue. Les travaux existants utilisent une approche eulérienne pour la phase continue et lagrangienne pour la phase dispersée. Ils ont pour objectif d'apporter des informations sur la fermeture des modèles moyennés. Cette approche est justifiée lorsque les fluctuations turbulentes à haute fréquence de la phase continue - filtrées par la SGE n'affectent pas la trajectoire des particules. Cette hypothèse est justifiée pour des particules lourdes (gaz-solide, liquidesolide). En écoulements à bulles où l'inertie plus faible des bulles lui fait amplifier les fluctuations à haute fréquence de la phase liquide, elle semble moins justifiée et doit encore démontrer son applicabilité à un domaine plus vaste.

L'approche de la simulation numérique directe (SND) avec suivi d'interfaces a beaucoup progressé ces dernières années. Cette approche permettra de véritables "expériences numériques" quand les limitations dues aux performances des ordinateurs mais également aux modèles physiques et aux méthodes numériques seront réduites. Dans le cas des écoulements sans changement de phase, elle permet soit de suivre plusieurs bulles [4], soit de prédire la déformation et le fractionnement d'une bulle, d'une goutte ou d'une interface en stratifié [5]. Certaines études autorisent même la prise en compte exacte du changement de phase (vaporisation, condensation), mais se limitent pour l'instant à des écoulements à faibles nombres Reynolds et pour de très petits domaines physiques $[6,7]$. Les études sont prometteuses, mais demandent encore à mûrir avant d'être appliquées de façon aussi intensive que les approches plus globales.

\section{- 3.2 Limitations des techniques numériques associées à la simulation}

L'approche classique de la conception des codes, s'appuyant essentiellement sur le langage Fortran, repose sur une analyse en terme de procédures : l'algorithme est une tâche précise où l'on fait appel à autant d'objets (tableaux, réels,...) que nécessaire à l'implémentation de cette tâche. L'inconvénient majeur de cette démarche est que l'on ne peut réutiliser que de façon très limitée les programmes générés, car ils sont dès le départ très spécifiques. On aboutit à une grande accumulation de procédures et de types de variables pourtant voisins. Les programmes deviennent rapidement longs et illisibles.

La taille des maillages dans les logiciels de thermohydraulique actuels est limitée à quelques millions d'éléments, mais les principales limitations résident essentiellement dans l'association d'un gros maillage avec une discrétisation très fine en temps, à la durée et à la complexité des transitoires étudiés. D'autres limitations proviennent de l'utilisation de volumes finis structurés qui ne permet pas le raffinement local ou la description de frontières complexes. Enfin, il n'est pas facile de passer d'un maillage de domaines simples et restreints à des applications réacteurs dont la géométrie est chargée d'obstacles.

\subsection{Limitations de l'instrumentation}

Les auteurs de cette communication invitent le lecteur à se reporter également à celle de Lemonnier [8] dédiée à l'instrumentation avancée. Membre de FASTNET, sa contribution complète la brève évocation réalisée ici dans ce domaine. Nous ne donnerons que quelques éléments des limitations de l'instrumentation regroupés arbitrairement en deux groupes : les méthodes intrusives, pour lesquelles il est nécessaire d'introduire une sonde dans l'écoulement, et les méthodes non intrusives, où l'information est obtenue sans interaction mécanique avec le milieu en résolvant, le cas échéant, un problème inverse de reconstruction.

Dans les méthodes intrusives applicables aux écoulements diphasiques, on peut citer :

- L'anémométrie à film chaud, pour caractériser les écoulements gaz-liquide où le liquide est la phase continue. Cette méthode permet de déterminer la vitesse moyenne locale, l'écart-type des fluctuations et le taux de présence moyen et local du liquide. La méthode exige un contrôle thermique rigoureux de l'installation expérimentale et reste limitée aux écoulements à direction privilégiée. A une exception près [9], cette méthode n'est pas utilisée en écoulement diphasique non adiabatique.

- Les sondes locales optiques ou électriques, qui permettent de déterminer la fonction indicatrice de phase et la vitesse d'interface par un traitement approprié des signaux provenant de plusieurs pointes voisines. L'étude du retard entre les signaux provenant des différentes pointes permet de déterminer la vitesse d'interface locale et, par moyenne temporelle, le temps de présence local et l'aire interfaciale locale. Les limitations de la technique proviennent de la nécessaire séparation des différentes pointes, qui peut raisonnablement être abaissée à une fraction de millimètre [10]. Cette séparation limite la taille des bulles détectables. De plus, la nature intrusive de la sonde réduit son application aux situations où la vitesse possède une direction privilégiée. Cette méthode est inutilisable pour des écoulements présentant des fluctuations de vitesse importantes en module et direction.

- Les sondes locales électromagnétiques, très simples de conception et d'emploi, qui permettent d'effectuer une mesure locale de vitesse dans un milieu au moins légèrement 
conducteur. Les limitations de cette technique locale résident, en attendant une future miniaturisation, dans la taille importante des capteurs et dans la bande passante limitée par des fréquences d'excitation de quelques centaines de $\mathrm{Hz}$.

Dans les méthodes non intrusives applicables aux écoulements diphasiques, on peut citer :

- L'anémométrie laser pour des taux de vide ne dépassant pas $7 \%$ et pour des écoulements à bulles dispersés et dilués [11].

- Les méthodes d'atténuation photoniques $\mathrm{X}$ ou $\mathrm{g}$ pour mesurer le taux de vide moyen sur un segment en écoulement diphasique. En multipliant les mesures sur différentes cordes, on peut reconstruire, par inversion tomographique, la distribution de taux de vide moyen dans une section de conduite. Cette technique éprouvée bénéficiera des progrès récents des détecteurs de rayons $\mathrm{X}$ à conversion directe et semble l'une des seules qui ne soit pas limitée dans son principe pour une application à des écoulements diphasiques quelconques, l'épaisseur de milieu traversée n'étant limitée que par la puissance de la source.

- Les méthodes ultrasonores pour déterminer en écoulement gaz-liquide ou liquide-liquide la fraction volumique de phase moyenne sur une corde d'une conduite. L'aire interfaciale et le taux de vide peuvent être d'ailleurs déterminés simultanément [12]. Ces techniques ont été analysées théoriquement en détail par Boyer [13] et leur utilisation n'est pour l'instant établie qu'en écoulement à bulles.

- La RMN dont l'utilisation a été montrée [14] pour déterminer simultanément le taux de vide moyen et les densités de probabilité des vitesses moyennes phasiques en écoulement gaz-liquide et liquide-liquide. Cette méthode est, pour l'instant, limitée au volume de mesure cylindrique d'une conduite.

\subsection{Limitations concernant les démarches expérimentales}

La démarche généralement utilisée en thermohydraulique face à un problème nouveau consiste à réaliser d'abord une ou plusieurs expériences de compréhension où l'on s'efforce d'identifier les phénomènes et de déterminer les paramètres pertinents. Ensuite, un programme à caractère analytique, où les phénomènes physiques sont si possible découplés (entre thermique, hydraulique et chimique) et où les paramètres varient de manière indépendante les uns par rapport aux autres, est construit. Dans cette première étape se pose la question du choix des techniques de mesure adaptées et de la gamme de paramètres à couvrir. Les limitations concernent essentiellement l'instrumentation utilisable et la restriction du domaine de paramètres à couvrir au domaine accessible par l'expérience. Par exemple, dans bien des cas d'expériences de crise d'ébullition, pour atteindre un titre thermodynamique donné, la géométrie de l'installation imposerait des conditions irréalistes (un écoulement d'eau en entrée avec une température inférieure à la solidification !).

Une fois les modèles ou corrélations ajustés et intégrés dans un code de calcul, il faut avoir recours à des expériences de validation globales couplant plusieurs phénomènes afin de s'assurer de la cohérence physique du modèle final. Les limitations de la pertinence de telles expériences sont souvent liées à des problèmes d'effet d'échelle (il peut être financièrement inacceptable de représenter le phénomène à l'échelle 1), des effets de distorsion de la géométrie et incluant l'utilisation de fluides de simulation. En revanche, sur de telles expériences, la mise en œuvre de techniques de mesures intrusives ou moins précises que celles utilisées pour l'étude fine d'un phénomène particulier peut être très utile pour une validation globale.

\section{IV — AXES DE DÉVELOPPEMENT}

\subsection{Propositions d'axes de recherche en modélisation physique}

Un important investissement a été fait sur la modélisation 1D des écoulements diphasiques ou polyphasiques, dans le cadre de développements de codes dédiés. Les modèles utilisés, les types de relation de fermeture, les méthodes numériques ont, suivant les cas, été très variés. Il apparaît nécessaire de réunir ce savoir-faire dans une base de connaissance, de rationaliser le développement et l'utilisation de ces outils 1D en jouant la carte de la réutilisabilité. Une plate-forme logicielle permettant d'utiliser les différents modèles, les relations de fermeture, les méthodes numériques, des fluides de nature différentes, répondrait à ces objectifs. Ses utilisations seraient multiples : interprétation d'expérience, essais de nouveaux modèles physiques ou de schémas numériques, base de développement pour des codes dédiés en réponse à des problèmes industriels ponctuels, préparations de modules 1D pour les futures générations de codes système. Un tel outil n'existe pas sur le marché. Audelà des éléments à regrouper, des acquisitions de connaissances concernent l'élaboration de modèles multi-champs et la prise en compte d'une équation de transport de l'aire interfaciale avec modélisation des termes de fractionnement, de coalescence, de nucléation, de condensation.

L'approche moyennée tridimensionnelle utilisant le modèle à deux fluides restera encore longtemps nécessaire, notamment pour des applications industrielles, en attendant que des modèles plus fins prennent le relais. C'est notamment la seule qui soit à la fois compatible avec les performances prévisibles des ordinateurs à moyen terme, et qui puisse aborder la totalité des régimes d'écoulement. Néanmoins, des efforts de modélisation sont à faire dans le domaine des écoulements déstructurés, peu exploré, mais d'une importance primordiale dans de nombreux problèmes industriels. Il est désormais également clair que des équations de transport supplémentaires sont nécessaires, telle l'équation de transport de l'aire interfaciale dont la connaissance permet notamment de définir la configuration locale de l'écoulement.

Les succès de la simulation des grandes échelles (SGE) en monophasique laissent espérer des progrès analogues dans le domaine diphasique. Néanmoins, en diphasique, aux fluctuations turbulentes qui peuvent s'étendre sur un large spectre, s'ajoutent les échelles liées à l'intermittence des phases qui peuvent couvrir une gamme encore plus large. Un filtrage de ces diverses échelles conduirait donc à simuler les grosses échelles turbulentes et à faire du suivi d'interfaces pour les grosses poches. Les petites échelles turbulentes et les petites structures diphasiques seraient moyennées et décrites par leurs effets sur les plus grosses. Les transferts entre champs simulé et filtré seraient multiples et dans les deux sens. La simulation des grandes échelles en diphasique cumule les difficultés de modélisation des approches à deux fluides moyennées et de suivi des interfaces, en y ajoutant celles liées aux transferts entre champs simulé et filtré. Cette voie est très importante mais délicate ; la stratégie suggérée est de s'appuyer sur la 
simulation numérique directe pour éclaircir les rapports entre champs simulés et moyennés de cette approche.

Dans la simulation numérique directe, des pas de discrétisation en espace et temps très petits sont requis pour prédire les plus petites échelles du problème. Cela limite l'application à des problèmes très réduits en dimension et nombre de Reynolds. Néanmoins l'obtention de champs locaux fluctuants peut servir à éclaircir des mécanismes locaux et, une fois la capacité de prédiction avérée, à construire des relations de fermetures locales pour des modèles plus globaux. La modélisation de l'ensemble d'un écoulement reste irréaliste.

\subsection{Propositions d'axes de recherche pour la simulation numérique}

La programmation orientée objet entre maintenant dans la conception des logiciels scientifiques. Elle offre la possibilité de réutiliser facilement, partiellement ou totalement, selon les besoins, des entités nommées "objet" ou "classes d'objets". Il devient simple d'enrichir la base des objets disponibles pour de nouvelles applications. L'architecture est ouverte. La réutilisabilité, la validité, la robustesse, l'extensibilité sont les principaux avantages de ce type de programmation. Le couplage entre codes de thermohydraulique et d'autres domaines de la physique est ainsi facilité, ainsi que l'utilisation de maillages non structurés.

\subsection{Propositions d'axes de recherche pour l'instrumentation}

Plusieurs présentations des axes de recherches sont possibles. L'accent peut être mis sur :

- les développements susceptibles d'améliorer la description d'une situation physique relative à un problème industriel spécifique, e.g., l'instrumentation en débit diphasique des tubes d'un échangeur à tubes et calandre.

- les développements susceptibles d'améliorer la détermination expérimentale d'une grandeur, indépendamment des applications, par exemple l'anémométrie thermique en écoulement non isotherme.

- les développements susceptibles de contribuer directement à l'amélioration des modèles d'écoulement diphasique comme par exemple l'application des techniques d'imagerie par résonance magnétique nucléaire pour la détermination des distributions de taux de vide en écoulement tridimensionnel.

A long terme, il semble justifié de privilégier les approches amont, et donc de favoriser les développements promettant des avancées significatives en matière de modélisation. Dans cette optique, il convient de coordonner les développements d'instrumentation aux besoins des modèles physiques à développer.

\subsection{Propositions d'axes de recherche concernant les démarches expérimentales}

Les démarches expérimentales devraient évoluer à long terme dans deux directions complémentaires : d'une part, des expériences très fondamentales pour l'étude et la quantification d'un phénomène local bien déterminé de taille relativement modeste (au niveau de la géométrie mais pas nécessairement au niveau des informations collectées) et d'autre part, des expériences de validation globales dont la taille peut être importante aussi bien au niveau de la géométrie que du nombre de mesures réalisées. Pour les expériences fondamentales, la recherche amènera des développements au niveau de l'instrumentation alors que pour les expériences globales, le travail concerne essentiellement les techniques expérimentales à mettre en œuvre. Cette mise en œuvre devra tenir compte des problèmes liés à la définition exacte du phénomène à étudier et des couplages à prendre en compte ou à éviter, la définition des paramètres pertinents et la justification des gammes à atteindre (et des gammes effectivement réalisées), la quantification des incertitudes de mesure et la forme finale des résultats (base de données).

Une place accrue devra être donnée aux expériences de visualisation directe (sections d'essais transparentes) utiles non seulement pour l'identification et la compréhension des phénomènes physiques, mais aussi pour leur rôle pédagogique, présentant les résultats sous forme compréhensible par des non-spécialistes.

\section{V — SYNTHÈSE DES PREMIÈRES RECOM- MANDATIONS DE LA FASTNET}

Les premières recommandations d'études de thermohydraulique proposées par la FASTNET sont basées sur une analyse des besoins du CEA et de l'industrie nucléaire et non nucléaire. Elles portent principalement sur le développement de modèles prédictifs validés. Le secteur aval a besoin d'outils de dimensionnement et de validation lui permettant de concevoir des équipements industriels pour de larges gammes de leurs paramètres de fonctionnement.

L'analyse de l'état de l'art des techniques disponibles a porté sur les quatre points suivants :

- la modélisation physique,

- les techniques expérimentales,

- l'instrumentation pour les écoulements diphasiques,

- les méthodes numériques.

L'analyse effectuée a permis de mettre à jour les limitations actuelles des différentes techniques et d'en identifier les verrous. Le groupe de travail est constitué de spécialistes en modélisation, techniques de mesure, techniques expérimentales et techniques numériques, ce qui confère aux conclusions une richesse résultant de leur effort de dialogue. L'expérience acquise lors du fonctionnement de la FASTNET montre qu'aucun de ces domaines n'a le monopole de la stratégie et qu'il y a, au contraire, intérêt à resserrer les liens, à tous niveaux, entre ces différentes branches d'activités complémentaires de la thermohydraulique.

Les recommandations actuelles de la FASTNET peuvent être synthétisées autour de projets comportant chacun des contributions au développement de modèles physiques appropriés, de techniques numériques, de techniques expérimentales et d'instrumentation afin de fournir à la fois la caractérisation des phénomènes préalable à l'élaboration des modèles et des données pour leur validation.

Ces recommandations doivent alimenter la réflexion sur les programmes de thermohydraulique monophasique et diphasique et stimuler la coopération entre unités du CEA, entre le CEA et ses partenaires habituels, et même si possible entre le CEA et d'autres partenaires concernés par les progrès envisagés. Trois axes ont été identifiés autour de trois préoccupations industrielles principales :

- un code monophasique tridimensionnel (hors du cadre du présent exposé),

- une plate-forme de développements de modèles diphasiques monodimensionnels, 
— une plate-forme de développements de modèles d'écoulements diphasiques tridimensionnels avec ou sans échanges de chaleur et de masse.

Chacun de ces axes est caractérisé par l'identification des besoins équilibrés d'effort de développements en matière de modèles physiques, d'expériences analytiques ou intégrales et d'analyse numérique. Plus particulièrement, les points suivants représentent des préoccupations communes aux 3 axes de recherches :

- Analyse des incertitudes liées (i) aux techniques expérimentales et à l'instrumentation, (ii) aux modèles physiques et, en particulier, aux constantes ajustables qu'ils contiennent et, (iii) à l'utilisation de techniques numériques de résolution des équations gouvernant un écoulement, en particulier les incertitudes liées à l'utilisation d'un schéma de discrétisation et leur séparation des incertitudes relatives aux modèles physiques.

- Obtention de solutions numériques de référence, permettant de séparer l'origine des incertitudes.

- Mise en cuvre des nouveaux langages de programmation, en particulier, les langages orientés objet.

- Construction et gestion de banques de données expérimentales cohérentes avec les objectifs de modélisation et de validation.

L'objet des développements est de faire reculer autant que possible la part d'empirisme encore présente dans les différents modèles de thermohydraulique diphasique et polyphasique. C'est la première étape indispensable qui ouvre la voie aux méthodes de simulation dont on ne peut que souhaiter à terme l'avènement, même s'il est probable que celui-ci demande encore quelques décennies avant de se traduire par la simulation complète d'applications industrielles à l'échelle de systèmes ou de composants.

\section{Références}

[1] Bestion D., Clément P., Garnier J., Hervieu E., lebaigue O. LEMONNIER H. ET VILLAND M., Mars 1997, Recommandations émises par le groupe FASTNET, Version 1, 82 p., Note CEA-Grenoble, DTP/ DIR 97-213, 3 mars 1997.
[2] Bestion D. Chément P. Garnier J., Caminade J.-P., Del haye J.-M. Dumaz P., Grand D., Hervieu E., Lebaigue O., Lemonnier h., Lhuillier C., Mercier P., Pages J.-R., Toumi I. et Vill.and M., 1998, Rapport FASTNET en préparation.

[3] MOREL C., 1996, An order of magnitude analysis of the two-phase $k-\varepsilon$ model, soumis à Fluid Mechanics Research.

[4] Esmaeel A. \& Tryggvason G., 1996, An inverse energy cascade in two-dimensional low Reynolds number bubbly flows, J. Fluid Mech., Vol. 314, pp. 315-330.

[5] Chen G., Kharif C., Zaleski S. \& Li J., 1996, Two-dimensional Navier-Stokes simulation of breaking waves, Soumis à Physics of Fluids.

[6] Jamet D., Lebaigue O., Coutris N. \& Del.haye J.M., 1995, A numerical description of a liquid-vapor interface based on the second gradient theory, Fluid Mechanics Research, Vol. 22, No. 1, pp. 1-14.

[7] JURIC D. \& TrYgGiason G., 1995, A front-tracking method for the liquid-vapor phase change, FED-Vol. 234, 1995 IMECE, pp. 141-148, Proc. of the ASME Fluids Engineering Division, ASME 1995.

[8] LEMONNIER H., 1998, Instrumentation avancée en écoulement diphasique : Rêve ou réalité ?, Colloque "Hydrodynamique des procédés industriels diphasiques".

[9] Shirat.Kar B.S. \& LAHEY R.T., 1972, Diabatic local void fraction measurement in Freon-114 with a hot-wire anemometer, ANS Trans., Vol. 15. No. 2, pp. 880-sqq.

[10] GARNIER J., 1997, Measurement of local two-phase flow pattern in boiling R/2 simulating PWR conditions with multiple optical probes, Proc. $\mathrm{OECD} / \mathrm{CSN}$ I Specialist Meeting on advanced instrumentation and measurement techniques, Santa Barbara, CA, March 17-20, 1997.

[11] LANCE M. \& BAtAlLLE J., 1991, Turbulence in the liquid phase of a uniform bubbly air-water flow, J. Fluid Mechanics, Vol. 222, pp. 95-118.

[12] Bensler H., 1990, Détermination de l'aire interfaciale, du taux de vide et du diamètre moyen de Sauter dans un écoulement à bulles à partir de l'atténuation d'un faisceau d'ultrasons, Doctorat INP-Grenoble.

[13] BOYER C., 1996, Etude d'un procédé de mesure des débits d'un écoulement triphasique eau-huile-gaz, Doctorat de I'Institut National Polytechnique de Grenoble.

[14] Javelot S., Leblond J. \& Baradel. C., 1995, Multiphase flow characterization by Nuclear Magnetic Resonance, Proc. of 2 nd Int. Conf. on Multiphase Flow, Serizawa, A., Fukano, T. and Bataille J., Eds, April 3-7. Kyoto, Vol. 1, IN2/33-38. 